Bull. Korean Math. Soc. 52 (2015), No. 2, pp. 593-601

http://dx.doi.org/10.4134/BKMS.2015.52.2.593

\title{
UNIVARIATE LEFT FRACTIONAL POLYNOMIAL HIGH ORDER MONOTONE APPROXIMATION
}

\author{
George A. Anastassiou
}

\begin{abstract}
Let $f \in C^{r}([-1,1]), r \geq 0$ and let $L^{*}$ be a linear left fractional differential operator such that $L^{*}(f) \geq 0$ throughout $[0,1]$. We can find a sequence of polynomials $Q_{n}$ of degree $\leq n$ such that $L^{*}\left(Q_{n}\right) \geq 0$ over $[0,1]$, furthermore $f$ is approximated left fractionally and simultaneously by $Q_{n}$ on $[-1,1]$. The degree of these restricted approximations is given via inequalities using a higher order modulus of smoothness for $f^{(r)}$
\end{abstract}

\section{Introduction}

The topic of monotone approximation started in [6] has become a major trend in approximation theory. A typical problem in this subject is: given a positive integer $k$, approximate a given function whose $k$ th derivative is $\geq 0$ by polynomials having this property.

In [3] the authors replaced the $k$ th derivative with a linear differential operator of order $k$. We mention this motivating result.

Theorem 1. Let $h, k, p$ be integers, $0 \leq h \leq k \leq p$ and let $f$ be a real function, $f^{(p)}$ continuous in $[-1,1]$ with modulus of continuity $\omega_{1}\left(f^{(p)}, x\right)$ there. Let $a_{j}(x), j=h, h+1, \ldots, k$ be real functions, defined and bounded on $[-1,1]$ and assume $a_{h}(x)$ is either $\geq$ some number $\alpha>0$ or $\leq$ some number $\beta<0$ throughout $[-1,1]$. Consider the operator

$$
L=\sum_{j=h}^{k} a_{j}(x)\left[\frac{d^{j}}{d x^{j}}\right]
$$

and suppose, throughout $[-1,1]$,

$$
L(f) \geq 0 .
$$

Received March 21, 2014

2010 Mathematics Subject Classification. 26A33, 41A10, 41A17, 41A25, 41A28, 41A29.

Key words and phrases. monotone approximation, Caputo fractional derivative, fractional linear differential operator, higher order modulus of smoothness. 
Then, for every integer $n \geq 1$, there is a real polynomial $Q_{n}(x)$ of degree $\leq n$ such that

$$
L\left(Q_{n}\right) \geq 0 \text { throughout }[-1,1]
$$

and

$$
\max _{-1 \leq x \leq 1}\left|f(x)-Q_{n}(x)\right| \leq C n^{k-p} \omega_{1}\left(f^{(p)}, \frac{1}{n}\right),
$$

where $C$ is independent of $n$ or $f$.

We use also the notation $I=[-1,1]$.

We would like to mention:

Theorem 2 (Gonska and Hinnemann [5]). Let $r \geq 0$ and $s \geq 1$. Then there exists a sequence $Q_{n}=Q_{n}^{(r, s)}$ of linear polynomial operators mapping $C^{r}(I)$ into $P_{n}$ (space of polynomials of degree $\leq n$ ), such that for all $f \in C^{r}(I)$, all $|x| \leq 1$ and all $n \geq \max (4(r+1), r+s)$ we have

(2) $\left|f^{(k)}(x)-\left(Q_{n} f\right)^{(k)}(x)\right| \leq M_{r, s}\left(\Delta_{n}(x)\right)^{r-k} \omega_{s}\left(f^{(r)}, \Delta_{n}(x)\right), 0 \leq k \leq r$,

where $\Delta_{n}(x)=\frac{\sqrt{1-x^{2}}}{n}+\frac{1}{n^{2}}$, and $M_{r, s}$ is a constant independent of $f, x$, and $n$. Above $\omega_{s}$ is the usual modulus of smoothnees of order $s$ with respect to the supremum norm.

Theorem 2 implies the useful:

Corollary $3([2])$. Let $r \geq 0$ and $s \geq 1$. Then there exists a sequence $Q_{n}=$ $Q_{n}^{(r, s)}$ of linear polynomial operators mapping $C^{r}(I)$ into $P_{n}$, such that for all $f \in C^{r}(I)$ and all $n \geq \max (4(r+1), r+s)$ we have

$$
\left\|f^{(k)}-\left(Q_{n} f\right)^{(k)}\right\|_{\infty} \leq \frac{C_{r, s}}{n^{r-k}} \omega_{s}\left(f^{(r)}, \frac{1}{n}\right), \quad k=0,1, \ldots, r,
$$

where $C_{r, s}$ is a constant independent of $f$ and $n$.

In [2] we proved the motivational:

Theorem 4. Let $h, v, r$ be integers, $0 \leq h \leq v \leq r$ and let $f \in C^{r}(I)$, with $f^{(r)}$ having modulus of smoothness $\omega_{s}\left(f^{(r)}, \delta\right)$ there, $s \geq 1$. Let $\alpha_{j}(x)$, $j=h, h+1, \ldots, v$ be real functions, defined and bounded on $I$ and suppose $\alpha_{h}$ is either $\geq \alpha>0$ or $\leq \beta<0$ throughout I. Take the operator

$$
L=\sum_{j=h}^{v} \alpha_{j}(x)\left[\frac{d^{j}}{d x^{j}}\right]
$$

and assume, throughout $I$,

$$
L(f) \geq 0 \text {. }
$$

Then for every integer $n \geq \max (4(r+1), r+s)$, there exists a real polynomial $Q_{n}(x)$ of degree $\leq n$ such that

$$
L\left(Q_{n}\right) \geq 0 \text { throughout } I,
$$


and

$$
\left\|f^{(k)}-Q_{n}^{(k)}\right\|_{\infty} \leq \frac{C}{n^{r-v}} \omega_{s}\left(f^{(r)}, \frac{1}{n}\right), \quad 0 \leq k \leq h .
$$

Moreover, we get

$$
\left\|f^{(k)}-Q_{n}^{(k)}\right\|_{\infty} \leq \frac{C}{n^{r-k}} \omega_{s}\left(f^{(r)}, \frac{1}{n}\right), \quad h+1 \leq k \leq r,
$$

were $C$ is a constant independent of $f$ and $n$.

In this article we extend Theorem 4 to the fractional level. Indeed here $L$ is replaced by $L^{*}$, a linear left Caputo fractional differential operator. Now the monotonicity property is only true on the critical interval [0,1]. Simultaneous and fractional convergence remains true on all of $I$.

We are also inspired by [1].

We make:

Definition 5 ([4], p. 50). Let $\alpha>0$ and $\lceil\alpha\rceil=m,(\lceil\cdot\rceil$ ceiling of the number $)$. Consider $f \in C^{m}([-1,1])$. We define the left Caputo fractional derivative of $f$ of order $\alpha$ as follows:

$$
\left(D_{*-1}^{\alpha} f\right)(x)=\frac{1}{\Gamma(m-\alpha)} \int_{-1}^{x}(x-t)^{m-\alpha-1} f^{(m)}(t) d t,
$$

for any $x \in[-1,1]$, where $\Gamma$ is the gamma function.

We set

$$
\begin{gathered}
D_{*-1}^{0} f(x)=f(x), \\
D_{*-1}^{m} f(x)=f^{(m)}(x), \quad \forall x \in[-1,1] .
\end{gathered}
$$

\section{Main result}

We present:

Theorem 6. Let $h, v, r$ be integers, $1 \leq h \leq v \leq r$ and let $f \in C^{r}([-1,1])$, with $f^{(r)}$ having modulus of smoothness $\omega_{s}\left(f^{(r)}, \delta\right)$ there, $s \geq 1$. Let $\alpha_{j}(x)$, $j=h, h+1, \ldots, v$ be real functions, defined and bounded on $[-1,1]$ and suppose $\alpha_{h}(x)$ is either $\geq \alpha>0$ or $\leq \beta<0$ on $[0,1]$. Let the real numbers $\alpha_{0}=0<$ $\alpha_{1} \leq 1<\alpha_{2} \leq 2<\cdots<\alpha_{r} \leq r$. Here $D_{*-1}^{\alpha_{j}} f$ stands for the left Caputo fractional derivative of $f$ of order $\alpha_{j}$ anchored at -1 . Consider the linear left fractional differential operator

$$
L^{*}:=\sum_{j=h}^{k} \alpha_{j}(x)\left[D_{*-1}^{\alpha_{j}}\right]
$$

and suppose, throughout $[0,1]$,

$$
L^{*}(f) \geq 0
$$


Then, for any $n \in \mathbb{N}$ such that $n \geq \max (4(r+1), r+s)$, there exists a real polynomial $Q_{n}(x)$ of degree $\leq n$ such that

$$
L^{*}\left(Q_{n}\right) \geq 0 \text { throughout }[0,1]
$$

and

$$
\begin{aligned}
& \sup _{-1 \leq x \leq 1}\left|\left(D_{*-1}^{\alpha_{j}} f\right)(x)-\left(D_{*-1}^{\alpha_{j}} Q_{n}\right)(x)\right| \\
\leq & \frac{2^{j-\alpha_{j}}}{\Gamma\left(j-\alpha_{j}+1\right)} \frac{C_{r, s}}{n^{r-j}} \omega_{s}\left(f^{(r)}, \frac{1}{n}\right)
\end{aligned}
$$

$j=h+1, \ldots, r ; C_{r, s}$ is a constant independent of $f$ and $n$.

Set

$$
l_{j}: \equiv \sup _{x \in[-1,1]}\left|\alpha_{h}^{-1}(x) \alpha_{j}(x)\right|, \quad h \leq j \leq v .
$$

When $j=1, \ldots, h$ we derive

$$
\begin{aligned}
& \sup _{-1 \leq x \leq 1}\left|\left(D_{*-1}^{\alpha_{j}} f\right)(x)-\left(D_{*-1}^{\alpha_{j}} Q_{n}\right)(x)\right| \leq \frac{C_{r, s}}{n^{r-v}} \omega_{s}\left(f^{(r)}, \frac{1}{n}\right) . \\
& {\left[\left(\sum_{\tau=h}^{v} l_{\tau} \frac{2^{\tau-\alpha_{\tau}}}{\Gamma\left(\tau-\alpha_{\tau}+1\right)}\right)\left(\sum_{\lambda=0}^{h-j} \frac{2^{h-\alpha_{j}-\lambda}}{\lambda ! \Gamma\left(h-\alpha_{j}-\lambda+1\right)}\right)+\frac{2^{j-\alpha_{j}}}{\Gamma\left(j-\alpha_{j}+1\right)}\right] .}
\end{aligned}
$$

Finally it holds

$$
\begin{aligned}
& \sup _{-1 \leq x \leq 1}\left|f(x)-Q_{n}(x)\right| \\
\leq & \frac{C_{r, s}}{n^{r-v}} \omega_{s}\left(f^{(r)}, \frac{1}{n}\right)\left[\frac{1}{h !} \sum_{\tau=h}^{v} l_{\tau} \frac{2^{\tau-\alpha_{\tau}}}{\Gamma\left(\tau-\alpha_{\tau}+1\right)}+1\right] .
\end{aligned}
$$

Proof. Here let $Q_{n}$ as in Corollary 3. Let $\alpha_{j}>0, j=1, \ldots, r$, such that $0<\alpha_{1} \leq 1<\alpha_{2} \leq 2<\alpha_{3} \leq 3<\cdots<\alpha_{r} \leq r$. That is $\left\lceil\alpha_{j}\right\rceil=j, j=1, \ldots, r$.

We consider the left Caputo fractional derivatives

$$
\left(D_{*-1}^{\alpha_{j}} f\right)(x)=\frac{1}{\Gamma\left(j-\alpha_{j}\right)} \int_{-1}^{x}(x-t)^{j-\alpha_{j}-1} f^{(j)}(t) d t,
$$

and

$$
\left(D_{*-1}^{j} f\right)(x)=f^{(j)}(x),
$$

and

$$
\begin{gathered}
\left(D_{*-1}^{\alpha_{j}} Q_{n}\right)(x)=\frac{1}{\Gamma\left(j-\alpha_{j}\right)} \int_{-1}^{x}(x-t)^{j-\alpha_{j}-1} Q_{n}^{(j)}(t) d t \\
\left(D_{*-1}^{j} Q_{n}\right)(x)=Q_{n}^{(j)}(x) ; j=1, \ldots, r .
\end{gathered}
$$

We notice that

$$
\left|\left(D_{*-1}^{\alpha_{j}} f\right)(x)-\left(D_{*-1}^{\alpha_{j}} Q_{n}\right)(x)\right|
$$




$$
\begin{aligned}
(20) & =\frac{1}{\Gamma\left(j-\alpha_{j}\right)}\left|\int_{-1}^{x}(x-t)^{j-\alpha_{j}-1} f^{(j)}(t) d t-\int_{-1}^{x}(x-t)^{j-\alpha_{j}-1} Q_{n}^{(j)}(t) d t\right| \\
& =\frac{1}{\Gamma\left(j-\alpha_{j}\right)}\left|\int_{-1}^{x}(x-t)^{j-\alpha_{j}-1}\left(f^{(j)}(t)-Q_{n}^{(j)}(t)\right) d t\right| \\
(21) & \leq \frac{1}{\Gamma\left(j-\alpha_{j}\right)} \int_{-1}^{x}(x-t)^{j-\alpha_{j}-1}\left|f^{(j)}(t)-Q_{n}^{(j)}(t)\right| d t \\
& \stackrel{(3)}{\leq} \frac{1}{\Gamma\left(j-\alpha_{j}\right)}\left(\int_{-1}^{x}(x-t)^{j-\alpha_{j}-1} d t\right) \frac{C_{r, s}}{n^{r-j}} \omega_{s}\left(f^{(r)}, \frac{1}{n}\right) \\
(22) & =\frac{1}{\Gamma\left(j-\alpha_{j}\right)} \frac{(x+1)^{j-\alpha_{j}}}{\left(j-\alpha_{j}\right)} \frac{C_{r, s}}{n^{r-j}} \omega_{s}\left(f^{(r)}, \frac{1}{n}\right) \\
& =\frac{(x+1)^{j-\alpha_{j}}}{\Gamma\left(j-\alpha_{j}+1\right)} \frac{C_{r, s}}{n^{r-j}} \omega_{s}\left(f^{(r)}, \frac{1}{n}\right) \\
& \leq \frac{2^{j-\alpha_{j}}}{\Gamma\left(j-\alpha_{j}+1\right)} \frac{C_{r, s}}{n^{r-j}} \omega_{s}\left(f^{(r)}, \frac{1}{n}\right) .
\end{aligned}
$$

We proved for any $x \in[-1,1]$ that

$$
\left|\left(D_{*-1}^{\alpha_{j}} f\right)(x)-\left(D_{*-1}^{\alpha_{j}} Q_{n}\right)(x)\right| \leq \frac{2^{j-\alpha_{j}}}{\Gamma\left(j-\alpha_{j}+1\right)} \frac{C_{r, s}}{n^{r-j}} \omega_{s}\left(f^{(r)}, \frac{1}{n}\right) .
$$

Hence it holds

$$
\sup _{-1 \leq x \leq 1}\left|\left(D_{*-1}^{\alpha_{j}} f\right)(x)-\left(D_{*-1}^{\alpha_{j}} Q_{n}\right)(x)\right| \leq \frac{2^{j-\alpha_{j}}}{\Gamma\left(j-\alpha_{j}+1\right)} \frac{C_{r, s}}{n^{r-j}} \omega_{s}\left(f^{(r)}, \frac{1}{n}\right),
$$

$j=0,1, \ldots, r$.

Above we set $D_{*-1}^{0} f(x)=f(x), D_{*-1}^{0} Q_{n}(x)=Q_{n}(x), \forall x \in[-1,1]$, and $\alpha_{0}=0$, i.e., $\left\lceil\alpha_{0}\right\rceil=0$.

Set also

$$
\rho_{n}:=C_{r, s} \omega_{s}\left(f^{(r)}, \frac{1}{n}\right)\left(\sum_{j=h}^{v} l_{j} \frac{2^{j-\alpha_{j}}}{\Gamma\left(j-\alpha_{j}+1\right)} n^{j-r}\right) .
$$

I. Suppose, throughout $[0,1], \alpha_{h}(x) \geq \alpha>0$. Let $Q_{n}(x), x \in[-1,1]$, be a real polynomial of degree $\leq n$ so that

$$
\begin{aligned}
& \max _{-1 \leq x \leq 1}\left|D_{*-1}^{\alpha_{j}}\left(f(x)+\rho_{n} \frac{x^{h}}{h !}\right)-\left(D_{*-1}^{\alpha_{j}} Q_{n}\right)(x)\right| \\
\leq & \frac{2^{j-\alpha_{j}}}{\Gamma\left(j-\alpha_{j}+1\right)} \frac{C_{r, s}}{n^{r-j}} \omega_{s}\left(f^{(r)}, \frac{1}{n}\right), j=0,1, \ldots, r .
\end{aligned}
$$


When $j=h+1, \ldots, r$, then

$$
\begin{aligned}
& \max _{-1 \leq x \leq 1}\left|\left(D_{*-1}^{\alpha_{j}} f\right)(x)-\left(D_{*-1}^{\alpha_{j}} Q_{n}\right)(x)\right| \\
\leq & \frac{2^{j-\alpha_{j}}}{\Gamma\left(j-\alpha_{j}+1\right)} \frac{C_{r, s}}{n^{r-j}} \omega_{s}\left(f^{(r)}, \frac{1}{n}\right),
\end{aligned}
$$

proving (14).

For $j=1, \ldots, h$ we get

$$
D_{*-1}^{\alpha_{j}}\left(\frac{x^{h}}{h !}\right)=\frac{1}{\Gamma\left(j-\alpha_{j}\right)} \int_{-1}^{x}(x-t)^{j-\alpha_{j}-1} \frac{t^{h-j}}{(h-j) !} d t
$$

(we see that $t=t+1-1$, and

$$
\begin{aligned}
t^{h-j}= & \left.((t+1)-1)^{h-j}=\sum_{\lambda=0}^{h-j}\left(\begin{array}{c}
h-j \\
\lambda
\end{array}\right)(t+1)^{h-j-\lambda}(-1)^{\lambda}\right) \\
= & \frac{1}{(h-j) ! \Gamma\left(j-\alpha_{j}\right)} \\
& \cdot \sum_{\lambda=0}^{h-j}(-1)^{\lambda}\left(\begin{array}{c}
h-j \\
\lambda
\end{array}\right) \int_{-1}^{x}(x-t)^{j-\alpha_{j}-1}(t+1)^{h-j-\lambda+1-1} d t \\
= & \frac{1}{(h-j) ! \Gamma\left(j-\alpha_{j}\right)} \\
& \cdot \sum_{\lambda=0}^{h-j}(-1)^{\lambda} \frac{(h-j) !}{\lambda !(h-j-\lambda) !} \frac{\Gamma\left(j-\alpha_{j}\right) \Gamma(h-j-\lambda+1)}{\Gamma\left(h-\alpha_{j}-\lambda+1\right)}(x+1)^{h-\alpha_{j}-\lambda} \\
(29)= & \sum_{\lambda=0}^{h-j} \frac{(-1)^{\lambda}}{\lambda ! \Gamma\left(h-\alpha_{j}-\lambda+1\right)}(x+1)^{h-\alpha_{j}-\lambda} .
\end{aligned}
$$

Hence for $j=1, \ldots, h$ we found that

$$
D_{*-1}^{\alpha_{j}}\left(\frac{x^{h}}{h !}\right)=\sum_{\lambda=0}^{h-j} \frac{(-1)^{\lambda}(x+1)^{h-\alpha_{j}-\lambda}}{\lambda ! \Gamma\left(h-\alpha_{j}-\lambda+1\right)} .
$$

Therefore we get from (26) that

$$
\begin{aligned}
& \max _{-1 \leq x \leq 1}\left|\left(D_{*-1}^{\alpha_{j}} f\right)(x)+\rho_{n}\left(\sum_{\lambda=0}^{h-j} \frac{(-1)^{\lambda}(x+1)^{h-\alpha_{j}-\lambda}}{\lambda ! \Gamma\left(h-\alpha_{j}-\lambda+1\right)}\right)-\left(D_{*-1}^{\alpha_{j}} Q_{n}\right)(x)\right| \\
\leq & \frac{2^{j-\alpha_{j}}}{\Gamma\left(j-\alpha_{j}+1\right)} \frac{C_{r, s}}{n^{r-j}} \omega_{s}\left(f^{(r)}, \frac{1}{n}\right), j=1, \ldots, h .
\end{aligned}
$$

Therefore we get for $j=1, \ldots, h$, that

$$
\max _{-1 \leq x \leq 1}\left|\left(D_{*-1}^{\alpha_{j}} f\right)(x)-\left(D_{*-1}^{\alpha_{j}} Q_{n}\right)(x)\right|
$$




$$
\begin{aligned}
(32) \leq & \rho_{n}\left(\sum_{\lambda=0}^{h-j} \frac{2^{h-\alpha_{j}-\lambda}}{\lambda ! \Gamma\left(h-\alpha_{j}-\lambda+1\right)}\right)+\frac{2^{j-\alpha_{j}}}{\Gamma\left(j-\alpha_{j}+1\right)} \frac{C_{r, s}}{n^{r-j}} \omega_{s}\left(f^{(r)}, \frac{1}{n}\right) \\
= & C_{r, s} \omega_{s}\left(f^{(r)}, \frac{1}{n}\right)\left(\sum_{\bar{j}=h}^{k} l_{\bar{j}} \frac{2^{\bar{j}-\alpha_{\bar{j}}}}{\Gamma\left(\bar{j}-\alpha_{\bar{j}}+1\right)} n^{\bar{j}-r}\right) \\
& \cdot\left(\sum_{\lambda=0}^{h-j} \frac{2^{h-\alpha_{j}-\lambda}}{\lambda ! \Gamma\left(h-\alpha_{j}-\lambda+1\right)}\right)+\frac{2^{j-\alpha_{j}}}{\Gamma\left(j-\alpha_{j}+1\right)} \frac{C_{r, s}}{n^{r-j}} \omega_{s}\left(f^{(r)}, \frac{1}{n}\right) \\
(33)= & C_{r, s} \omega_{s}\left(f^{(r)}, \frac{1}{n}\right)\left[\left(\sum_{\bar{j}=h}^{k} l_{\bar{j}} \frac{2^{\bar{j}-\alpha_{\bar{j}}}}{\Gamma\left(\bar{j}-\alpha_{\bar{j}}+1\right)} \frac{1}{n^{r-\bar{j}}}\right)\right. \\
& \left.\cdot\left(\sum_{\lambda=0}^{h-j} \frac{2^{h-\alpha_{j}-\lambda}}{\lambda ! \Gamma\left(h-\alpha_{j}-\lambda+1\right)}\right)+\frac{2^{j-\alpha_{j}}}{\Gamma\left(j-\alpha_{j}+1\right)} \frac{1}{n^{r-j}}\right] \\
\leq & C_{r, s} \omega_{s}\left(f^{(r)}, \frac{1}{n}\right) \frac{1}{n^{r-v}}\left[\left(\sum_{\bar{j}=h}^{v} l_{\bar{j}} \frac{2^{\bar{j}-\alpha_{\bar{j}}}}{\Gamma\left(\bar{j}-\alpha_{\bar{j}}+1\right)}\right)\right. \\
& \left.\cdot\left(\sum_{\lambda=0}^{h-j} \frac{2^{h-\alpha_{j}-\lambda}}{\lambda ! \Gamma\left(h-\alpha_{j}-\lambda+1\right)}\right)+\frac{2^{j-\alpha_{j}}}{\Gamma\left(j-\alpha_{j}+1\right)}\right] .
\end{aligned}
$$

Hence for $j=1, \ldots, h$ we derived (16):

$$
\max _{-1 \leq x \leq 1}\left|\left(D_{*-1}^{\alpha_{j}} f\right)(x)-\left(D_{*-1}^{\alpha_{j}} Q_{n}\right)(x)\right| \leq \frac{C_{r, s}}{n^{r-v}} \omega_{s}\left(f^{(r)}, \frac{1}{n}\right) .
$$

$$
\left[\left(\sum_{\tau=h}^{v} l_{\tau} \frac{2^{\tau-\alpha_{\tau}}}{\Gamma\left(\tau-\alpha_{\tau}+1\right)}\right)\left(\sum_{\lambda=0}^{h-j} \frac{2^{h-\alpha_{j}-\lambda}}{\lambda ! \Gamma\left(h-\alpha_{j}-\lambda+1\right)}\right)+\frac{2^{j-\alpha_{j}}}{\Gamma\left(j-\alpha_{j}+1\right)}\right] .
$$

From (26) when $j=0$ we obtain

$$
\max _{-1 \leq x \leq 1}\left|f(x)+\rho_{n} \frac{x^{h}}{h !}-Q_{n}(x)\right| \leq \frac{C_{r, s}}{n^{r}} \omega_{s}\left(f^{(r)}, \frac{1}{n}\right) .
$$

And

$$
\begin{aligned}
& \max _{-1 \leq x \leq 1}\left|f(x)-Q_{n}(x)\right| \leq \frac{\rho_{n}}{h !}+\frac{C_{r, s}}{n^{r}} \omega_{s}\left(f^{(r)}, \frac{1}{n}\right) \\
= & \frac{C_{r, s}}{h !} \omega_{s}\left(f^{(r)}, \frac{1}{n}\right)\left(\sum_{\tau=h}^{v} l_{\tau} \frac{2^{\tau-\alpha_{\tau}}}{\Gamma\left(\tau-\alpha_{\tau}+1\right)} n^{\tau-r}\right)+\frac{C_{r, s}}{n^{r}} \omega_{s}\left(f^{(r)}, \frac{1}{n}\right) \\
= & C_{r, s} \omega_{s}\left(f^{(r)}, \frac{1}{n}\right)\left[\frac{1}{h !} \sum_{\tau=h}^{v} l_{\tau} \frac{2^{\tau-\alpha_{\tau}}}{\Gamma\left(\tau-\alpha_{\tau}+1\right) n^{r-\tau}}+\frac{1}{n^{r}}\right]
\end{aligned}
$$


(38) $\leq \frac{C_{r, s}}{n^{r-v}} \omega_{s}\left(f^{(r)}, \frac{1}{n}\right)\left[\frac{1}{h !} \sum_{\tau=h}^{k} l_{\tau} \frac{2^{\tau-\alpha_{\tau}}}{\Gamma\left(\tau-\alpha_{\tau}+1\right)}+1\right]$,

that is proving (17).

Also if $0 \leq x \leq 1$, then

$$
\begin{aligned}
& \alpha_{h}^{-1}(x) L^{*}\left(Q_{n}(x)\right) \\
= & \alpha_{h}^{-1}(x) L^{*}(f(x))+\rho_{n} \frac{(x+1)^{h-\alpha_{h}}}{\Gamma\left(h-\alpha_{h}+1\right)} \\
& +\sum_{j=h}^{v} \alpha_{h}^{-1}(x) \alpha_{j}(x)\left[D_{*-1}^{\alpha_{j}} Q_{n}(x)-D_{*-1}^{\alpha_{j}} f(x)-\frac{\rho_{n}}{h !} D_{*-1}^{\alpha_{j}} x^{h}\right] \\
\stackrel{(26)}{\geq} & \rho_{n} \frac{(x+1)^{h-\alpha_{h}}}{\Gamma\left(h-\alpha_{h}+1\right)}-\left(\sum_{j=h}^{v} l_{j} \frac{2^{j-\alpha_{j}}}{\Gamma\left(j-\alpha_{j}+1\right)} \frac{C_{r, s}}{n^{r-j}} \omega_{s}\left(f^{(r)}, \frac{1}{n}\right)\right) \\
(40)= & \rho_{n} \frac{(x+1)^{h-\alpha_{h}}}{\Gamma\left(h-\alpha_{h}+1\right)}-\rho_{n}=\rho_{n}\left[\frac{(x+1)^{h-\alpha_{h}}}{\Gamma\left(h-\alpha_{h}+1\right)}-1\right] \\
(41)= & \rho_{n}\left[\frac{(x+1)^{h-\alpha_{h}}-\Gamma\left(h-\alpha_{h}+1\right)}{\Gamma\left(h-\alpha_{h}+1\right)}\right] \geq \rho_{n}\left[\frac{1-\Gamma\left(h-\alpha_{h}+1\right)}{\Gamma\left(h-\alpha_{h}+1\right)}\right] \geq 0 .
\end{aligned}
$$

Explanation: We know that $\Gamma(1)=1, \Gamma(2)=1$, and $\Gamma$ is convex and positive on $(0, \infty)$. Here $0 \leq h-\alpha_{h}<1$ and $1 \leq h-\alpha_{h}+1<2$. Thus $\Gamma\left(h-\alpha_{h}+1\right) \leq 1$ and $1-\Gamma\left(h-\alpha_{h}+1\right) \geq 0$. Hence $L^{*}\left(Q_{n}(x)\right) \geq 0, x \in[0,1]$.

II. Suppose on $[0,1]$ that $\alpha_{h}(x) \leq \beta<0$. Let $Q_{n}(x), x \in[-1,1]$, be a real polynomial of degree $\leq n$ so that

$$
\begin{aligned}
& \max _{-1 \leq x \leq 1}\left|D_{*-1}^{\alpha_{j}}\left(f(x)-\rho_{n} \frac{x^{h}}{h !}\right)-\left(D_{*-1}^{\alpha_{j}} Q_{n}\right)(x)\right| \\
\leq & \frac{2^{j-\alpha_{j}}}{\Gamma\left(j-\alpha_{j}+1\right)} \frac{C_{r, s}}{n^{r-j}} \omega_{s}\left(f^{(r)}, \frac{1}{n}\right), j=0,1, \ldots, r .
\end{aligned}
$$

Similarly we obtain again inequalities of convergence, see (14), (16) and (17).

Also if $0 \leq x \leq 1$, then

$$
\begin{aligned}
& \alpha_{h}^{-1}(x) L^{*}\left(Q_{n}(x)\right) \\
= & \alpha_{h}^{-1}(x) L^{*}(f(x))-\rho_{n} \frac{(x+1)^{h-\alpha_{h}}}{\Gamma\left(h-\alpha_{h}+1\right)} \\
& +\sum_{j=h}^{v} \alpha_{h}^{-1}(x) \alpha_{j}(x)\left[D_{*-1}^{\alpha_{j}} Q_{n}(x)-D_{*-1}^{\alpha_{j}} f(x)+\frac{\rho_{n}}{h !}\left(D_{*-1}^{\alpha_{j}} x^{h}\right)\right] \\
\stackrel{(42)}{\leq}- & \rho_{n} \frac{(x+1)^{h-\alpha_{h}}}{\Gamma\left(h-\alpha_{h}+1\right)}+\sum_{j=h}^{v} l_{j} \frac{2^{j-\alpha_{j}}}{\Gamma\left(j-\alpha_{j}+1\right)} \frac{C_{r, s}}{n^{r-j}} \omega_{s}\left(f^{(r)}, \frac{1}{n}\right)
\end{aligned}
$$




$$
\begin{aligned}
& \text { (44) } \quad=\rho_{n}\left(1-\frac{(x+1)^{h-\alpha_{h}}}{\Gamma\left(h-\alpha_{h}+1\right)}\right)=\rho_{n}\left(\frac{\Gamma\left(h-\alpha_{h}+1\right)-(x+1)^{h-\alpha_{h}}}{\Gamma\left(h-\alpha_{h}+1\right)}\right) \\
& \text { (45) } \leq \rho_{n}\left(\frac{1-(x+1)^{h-\alpha_{h}}}{\Gamma\left(h-\alpha_{h}+1\right)}\right) \leq 0,
\end{aligned}
$$

and hence on $[0,1]$ again holds $L^{*}\left(Q_{n}(x)\right) \geq 0$.

Remark 7 (to Theorem 6). Suppose that $\alpha_{j}(x), j=h, h+1, \ldots, v$ are continuous functions on $[-1,1]$, and we have on $[0,1]$ only $L^{*}(f)>0$. Relax the condition $\alpha_{h}(x)$ is either $\geq \alpha>0$ or $\leq \beta<0$ on $[0,1]$. Let $Q_{n}$ be the polynomial of degree $\leq n$ corresponding to $f$ from (24).

Then $D_{*-1}^{\alpha_{j}} Q_{n}$ converges uniformly to $D_{*-1}^{\alpha_{j}} f$ at a higher rate given by inequality (24), in particular for $0 \leq j \leq h$. Moreover, because $L^{*}\left(Q_{n}\right)$ converges uniformly to $L^{*}(f)$ on $[-1,1], L^{*}\left(Q_{n}\right)>0$ on $[0,1]$ for sufficiently large $n$.

\section{References}

[1] G. A. Anastassiou, Bivariate Monotone Approximation, Proc. Amer. Math. 112 (1991), no. 4, 959-964.

[2], Higher order monotone approximation with linear differential operators, Indian J. Pure Appl. Math. 24 (1993), no. 4, 263-266.

[3] G. A. Anastassiou and O. Shisha, Monotone approximation with linear differential operators, J. Approx. Theory 44 (1985), no. 4, 391-393.

[4] K. Diethelm, The Analysis of Fractional Differential Equations, Lecture Notes in Mathematics, Vol. 2004, 1st edition, Springer, New York, Heidelberg, 2010.

[5] H. H. Gonska and E. Hinnemann, Pointwise estimates for approximation by algebraic polynomials, Acta Math. Hungar. 46 (1985), no. 3-4, 243-254.

[6] O. Shisha, Monotone approximation, Pacific J. Math. 15 (1965), 667-671.

Department of Mathematical Sciences

UNIVERSITY OF MEMPHIS

MEMPhis, TN 38152, USA

E-mail address: ganastss@memphis.edu 